\title{
The Euler number of hyper-Kähler manifolds of OG10 type
}

\author{
Klaus Hulek ${ }^{\mathbb{D}}$, Radu Laza $^{\mathbb{D}}$, Giulia Saccà $^{\mathbb{D}}$
}

\begin{abstract}
Using the [21] construction, we give a simple proof for the fact that the Euler characteristic of a hyper-Kähler manifold of OG10 type is $\chi(\mathrm{OG} 10)=176,904$, a result previously established by Mozgovoy $[22]$.
\end{abstract}

\section{Introduction}

Algebraic manifolds with trivial canonical bundle, or more generally Ricci-flat compact Kähler manifolds, are an important class of manifolds and play a special role in the classification of algebraic varieties. By the famous decomposition theorem of Beauville and Bogomolov [2], Ricci flat compact Kähler manifolds are, up to finite cover, products of tori, Calabi-Yau manifolds (CY) and hyper-Kähler manifolds (HK), the latter also known as irreducible holomorphic symplectic manifolds (IHSM). So

2020 AMS Subject Classification: 14J20, 14D06.

Key Words and Phrases: Hyperkähler manifolds, abelian fibrations, Prym varieties, Euler characteristic.

First author is grateful to DFG for partial support under grant $\mathrm{Hu} 337 / 7-1$, the second author is supported in part by NSF grants DMS-1254812 and DMS-1802128, the third author is supported in part by NSF grant DMS-1801818. The last two authors wish to thank Leibniz Universität Hannover for the warm hospitality and good working conditions during their visits. 
far only very few examples of hyper-Kähler manifolds are known: these are two infinite series (with one case in each even dimension) namely manifolds which are deformation equivalent to Hilbert schemes of points on $K 3$ surfaces and so-called generalized Kummer varieties, together with two sporadic examples in dimension 6 and 10 respectively, due to O'Grady (denoted OG6 and OG10 below). It is a basic question to understand the topology (e.g. the Betti numbers) of these manifolds. The two infinite series are closely related to symmetric powers of $K 3$ surfaces and abelian surfaces respectively, leading to a full description of their cohomology rings (e.g. $[13,14]$ ). The topology of the six dimensional O'Grady example was determined by the third author and her collaborators [23]. The purpose of this note is to give a simple proof for the computation of the topological Euler characteristic for OG10, a result first established in the thesis of S. Mozgovoy [22].

Theorem 1.1. The Euler characteristic for a hyper-Kähler manifold of OG10 type is 176,904 .

An obvious natural question is to determine the Betti (and Hodge) numbers of hyper-Kähler manifolds $Z$ of OG10 type. This will be discussed elsewhere $^{1}$. For now, we only note the following known (mostly general) restrictions on the Betti numbers. Clearly, by definition, $b_{1}(Z)=0$ for all hyper-Kähler manifolds. Also, the second Betti number has been computed for all known examples of HK. In particular, the case of OG10 was done by Rapagnetta [26], who showed that $b_{2}(Z)=24$. By Verbitsky [30] (see also [20]), it is also known that for any HK $Z$ of dimension $2 n$, and any $k \in\{2, \ldots, n\}$, the cup product defines a natural inclusion $\operatorname{Sym}^{k} H^{2}(Z, \mathbb{Z}) \hookrightarrow H^{2 k}(Z, \mathbb{Z})$, and thus $b_{2 k} \geq\left(\begin{array}{c}b_{2}+k-1 \\ k\end{array}\right)$. Salamon [29] proved that

$$
2 \sum_{l=1}^{2 n}(-1)^{l}\left(3 l^{2}-n\right) b_{2 n-l}=n b_{2 n} .
$$

\footnotetext{
${ }^{1}$ (Note added in proof) This was now settled in [9]. Subsequently, further information on the cohomology of hyper-Kähler manifolds of OG10 type was obtained in [12].
} 
Together with the knowledge of the Euler number, these relations give some strong restrictions on the Betti numbers, but not sufficient to determine them for hyper-Kähler manifolds of OG10 type.

Our argument for the computation of $\chi(Z)$ is an adaptation of Beauville's method for counting curves on a K3 surface [3] which extends the standard computation of the Euler number for $K 3$ surfaces by counting the number of singular fibers in an elliptic $K 3$ surface. Similarly, our starting point is the construction of [21] for hyper-Kähler manifolds $Z$ of OG10 type as Lagrangian fibrations $Z=\overline{\mathcal{J}} \rightarrow B$ associated to a cubic fourfold $X \subset \mathbb{P}^{5}$. Namely, the general fiber $\mathcal{J}_{b}$ is the intermediate Jacobian of the cubic threefold $Y_{b}=X \cap H_{b}$ where $H_{b}$ is the hyperplane corresponding to a point $b \in B:=\left(\mathbb{P}^{5}\right)^{\vee}$. This leads, as originally observed in [10], to an open Lagrangian fibration $\mathcal{J} / U$ over the smooth locus $U=B \backslash X^{\vee}$ (with $\left.\mathcal{J}=\overline{\mathcal{J}}_{\mid U}\right)$. On the other hand, by a result of Mumford [24] the intermediate Jacobian $\mathcal{J}_{b}$ is isomorphic to a Prym variety. Using this description, in [21], the compactification $Z=\overline{\mathcal{J}}$ was then constructed, étale locally, as a relative compactified Prym over $B$. Returning to the proof of Theorem 1.1, by standard arguments, recalled in Section 2, it will be enough to consider only the fibers with $\chi\left(\overline{\mathcal{J}}_{b}\right) \neq 0$ (Cor. 2.2), which are only finitely many. Now, by construction, the discriminant in $B$ of $\overline{\mathcal{J}} / B$ is the dual variety $X^{\vee}$, which is naturally stratified in terms of singularities of the tangent hyperplanes to $X$ (i.e. the singularities of the cubic threefold $X \cap H)$. For a point in the discriminant, the associated limit compactified intermediate Jacobian has non-zero Euler characteristic only if it has no abelian factor, which in turn is equivalent, under the assumption that $X$ is generic, to saying that $H$ is a 5 -tangent hyperplane to $X$. As in the case of very general elliptically fibered $K 3$ s, the contribution (of the 5 -tangent hyperplane sections) to the Euler characteristic is 1 (Cor. 3.4). Thus the computation of the Euler characteristic reduces to the enumerative question of counting the number of 5-tangent hyperplanes to a general cubic fourfold. These type of questions (and much more) are answered by the theory of Thom polynomials of singularities (e.g. Rimányi [28], 
Kazarian [16]).

At this point we would like to compare our approach to that of Mozgovoy [22]. His starting point goes back to O'Grady [25] (see also [26]) who first constructed hyper-Kähler manifolds of OG10 type as symplectic resolutions $\widetilde{M}$ of certain moduli spaces $M$ of sheaves on $K 3$ surfaces $S$. Namely, applying O'Grady's construction to the case of polarized $K 3$ surfaces $(S, H)$ of degree 2 , one notes that the linear system $|2 H|$ has dimension 5 and then by associating to each sheaf its Fitting support, one obtains a fibration $\widetilde{M} \rightarrow|2 H|$ whose fibers are themselves moduli spaces of sheaves on (possibly singular) curves $C \in|2 H|$. Similar to the argument that we use here, Mozgovoy then uses this fibration and the additivity of the Euler number to compute $\chi(\widetilde{M})$. However, this is technically somewhat involved as the curve $C$ can be singular, reducible and even non-reduced. Consequently, one needs to keep track of the Euler numbers for various special fibers. In contrast, in our situation there is only one type of relevant special fiber, which comes with multiplicity 1 and Euler characteristic 1. Furthermore, one can view Mozgovoy's computation as a degeneration of our computation. This is due to the fact that cubic fourfolds degenerate to the secant variety of the Veronese surface in $\mathbb{P}^{5}$; the limit mixed Hodge structure associated to such a 1-parameter degeneration is pure, and, in fact, can be naturally identified with the Hodge structure of a degree $2 K 3$ surface $S$ (see [18]). Keeping track of the associated [21] fibration as the cubic fourfold $X$ degenerates to the secant variety of the Veronese surface, one recovers the original O'Grady construction associated to $S$ as described above (see [17, §5.3] for details). Finally, in the limit, the 5 -tangent hyperplanes that we consider lead to the special curves that enter into Mozgovoy's computation. In other words, the locus $\mathfrak{L}$ of Lagrangian fibered OG10 manifolds constructed by [21] is 20-dimensional, giving a Noether-Lefschetz divisor in the moduli space $\mathfrak{M}$ of polarized OG10 hyper-Kähler manifolds, while the locus $\mathfrak{L}^{\prime}$ obtained by O'Grady's method starting with a degree $2 K 3$ surface $S$ is 19-dimensional. The argument sketched above says that $\mathfrak{L}^{\prime} \subset \mathfrak{L}$ (and, in fact, a divisor), 
showing that indeed one can regard Mozgovoy's computation as a limit of ours.

\section{The Prym construction of OG10 and enumer- ative geometry}

\section{$2.1 \quad$ Preliminaries}

We recall that the Euler characteristic for algebraic varieties satisfies $\chi(Z)=\chi_{c}(Z)\left(:=\sum(-1)^{i} \operatorname{dim}_{\mathbb{Q}} H_{c}^{i}(Z, \mathbb{Q})\right)$ (e.g. [11, p. 141]). Consequently, the Euler characteristic is additive with respect to open and closed embeddings, i.e. for $W \subset Z$ closed, and $U=Z \backslash W$, we have $\chi(Z)=\chi(W)+\chi(U)$. Furthermore, the Euler characteristic is multiplicative for smooth proper fibrations of algebraic varieties. In particular, in our set-up: fibrations in complex tori - the Euler characteristic for the smooth part is 0 (e.g. $\chi(\mathcal{J})=0$, where $\mathcal{J} / U$ is as in the introduction). Thus, it remains to consider the behavior of the fibration $\overline{\mathcal{J}} / B$ over the singular part. In fact, by considering a Whitney stratification, one can show that only the fibers with non-zero Euler characteristic are relevant for the computation. Moreover, it turns out that there is only a finite number of them. Specifically, the following holds.

Proposition 2.1. Let $Z^{\circ} \rightarrow B^{\circ}$ be a proper morphism of complex algebraic varieties such that $\chi\left(Z_{b}\right)=0$ for all $b \in B^{\circ}$, then $\chi(Z)=0$.

Proof. This is a particular case of [8, Prop. 2.4] which gives a general "multiplicative" formula for the Euler characteristic for a proper map of algebraic varieties (in terms of a Whitney stratification).

From this, we conclude:

Corollary 2.2. Let $Z \rightarrow B$ be a proper surjective morphism, and $\Sigma_{0}$ be a finite set such that $\chi\left(Z_{b}\right)=0$ for $b \in B \backslash \Sigma_{0}$. Then $\chi(Z)=\sum_{b \in \Sigma_{0}} \chi\left(Z_{b}\right)$. 
As already mentioned, we will apply this result to the Lagrangian fibration $Z=\overline{\mathcal{J}} / B$ constructed in [21] as a model for OG10 HK manifolds (we note that the locus of Lagrangian fibered OG10 is a codimension 1 locus in moduli). Below, we review this construction and discuss the relevant stratification of the discriminant.

\subsection{The [21] construction of OG10}

Let $X \subset \mathbb{P}^{5}$ be a general cubic fourfold, and let $B:=\left(\mathbb{P}^{5}\right)^{\vee}$ be the projective space parameterizing its hyperplane sections. We denote by $U \subset\left(\mathbb{P}^{5}\right)^{\vee}$ the open locus parameterizing smooth hyperplane sections. A hyperplane section $Y_{b}=X \cap H_{b}$ for $b \in U$ is a smooth cubic threefold, whose associated intermediate Jacobian $J\left(Y_{b}\right)$ is a principally polarized abelian variety of dimension 5 (cf. [4]). Considering the family of such intermediate Jacobians leads to a morphism of quasi-projective varieties $\pi_{U}: \mathcal{J} \rightarrow U$, and furthermore $\mathcal{J}$ carries a holomorphic symplectic form, with respect to which $\mathcal{J} / U$ is a Lagrangian fibration (see [10, §8.5.2]). The content of [21] is the construction of a smooth compactification $\overline{\mathcal{J}} / B$ of $\mathcal{J} / U$ such that the holomorphic form extends and remains non-degenerate.

Theorem 2.3 (Laza-Saccà-Voisin [21]). Let $X$ be a general cubic fourfold. There exists a smooth projective compactification $Z=\overline{\mathcal{J}}$ of $\mathcal{J}$, which is a hyper-Kähler manifold and such that $\pi_{U}$ extends to a Lagrangian fibration $\pi: \overline{\mathcal{J}} \rightarrow B$. Moreover, $Z=\overline{\mathcal{J}}$ is of OG10 type.

As discussed above, in order to prove Theorem 1.1, we need to understand the singular fibers of $\overline{\mathcal{J}} / B$ and their Euler characteristic. This is closely related to the study of degenerations of intermediate Jacobians (see esp. [5,6]). The main tool for studying degenerations of intermediate Jacobians is Mumford's description of the intermediate Jacobian as a Prym variety. Specifically, if $Y$ is a smooth cubic threefold, and $\ell \subset Y$ is a general line, then the projection from $\ell$ realizes $\widetilde{Y}:=\mathrm{Bl}_{\ell} Y$ as a conic bundle over $\mathbb{P}^{2}$. The discriminant of $\widetilde{Y} \rightarrow \mathbb{P}^{2}$ is a quintic curve $C$, and furthermore $\tilde{Y} \rightarrow \mathbb{P}^{2}$ naturally determines an étale double cover $D \rightarrow C$. 
Mumford's theorem then says that $J(Y) \cong \operatorname{Prym}(D, C)$. Based on earlier results of Beauville, Casalaina-Martin and Laza [6] have noticed that the Prym construction also works well in the singular case (for $Y$ mildly singular), as long as one makes a careful choice of the line $\ell$. Furthermore, if $Y$ is any hyperplane section of a (Hodge) general cubic fourfold $X$, then $\ell$ can be chosen to be a "very good line" (see [21, Def. 2.9]). In short, the relevant statement for us is the following:

Proposition 2.4 ( [21, Prop. 2.3], [6]). Let $X$ be a general cubic fourfold. Then for any hyperplane section $Y=X \cap H$ there exists a line $\ell \subset Y$ such that

1. The double cover $f: D \rightarrow C$ associated to the conic bundle $\tilde{Y} \rightarrow \mathbb{P}^{2}$ is étale and both curves $D$ and $C$ are irreducible;

2. The singularities of $Y$ and those of $C$ are in one-to-one correspondence, including the analytic type (i.e. there is a bijection $\varphi$ : $\operatorname{Sing}(Y) \rightarrow \operatorname{Sing}(C)$, and the germ $(Y, y)$ is a double suspension of $(C, \varphi(y)))$.

Remark 2.5. A key fact about the hyperplane sections of a general cubic fourfold $X$ is that the linear system of hyperplane sections of $X$ gives a simultaneous versal deformation of the singularities of any hyperplane section $Y$ of $X$ (see [21, Prop. 3.6]; see also [5, Sect. 3] for a related discussion). The same is true for the associated curves $C$. More precisely, given $Y$ and a choice of very good line $\ell$, one gets a (possibly singular) quintic $C$. A small embedded deformation of $(Y, \ell)$ (in $X$ ) determines a family of quintics, which versally deform the singularities of $C$. In particular, this bounds the Milnor number of the singularities of $C$, and by standard singularity theory, it follows that only the types $A_{1}, \ldots, A_{5}, D_{4}, D_{5}$ can occur. Finally, property (2) above says that $C$ has the same number and type of $A_{i}$ and $D_{k}$ singularities as $Y$.

Returning to the [21] construction of $\overline{\mathcal{J}} / B$, we note that locally, in the étale or analytic topology, $\overline{\mathcal{J}} / B$ is the relative compactified Prym 
associated to a family of curves $(\mathcal{D}, \mathcal{C})$ which is obtained via projection from a (local) family of good lines on the universal family of hyperplane sections $\mathcal{Y} / B$ of $X$. By [21, Prop. 5.1 and Thm. 5.7] the fiber of $\pi: \overline{\mathcal{J}} \rightarrow$ $B$ over $b \in B$ is the compactified Prym variety of a double cover $D \rightarrow C$ of irreducible locally planar curves, i.e.

$$
\overline{\mathcal{J}}_{b}=\overline{\operatorname{Prym}}(D, C)=: \bar{P}_{D / C} \cdot
$$

(We note that since both $C$ and $D$ are irreducible with planar singularities, there is no ambiguity in the definition of the compactified Prym). We recall that the compactified Prym is a natural adaptation (in the double cover set-up) of the compactified Jacobian. We refer to [21, §4] and to Section 3 for the relevant notation, definitions and first properties of compactified Prym varieties. For the moment, we recall that the compactified Prym variety has an abelian variety factor, namely the "compact part" of the generalized Prym variety of $D$ over $C$ (see Section 3 for the relevant definitions). As discussed in Section 3 below (based on ideas from [3]), the relevant case for us is when this abelian factor vanishes. Under the étale assumption, this is equivalent to saying that the genus of the normalization of $C$ is 1 , a case that is described geometrically below.

Lemma 2.6. Let $C$ be a plane quintic with a combination of $A_{l}$ and $D_{k}$ singularities, denoted $\sum_{l} m_{l} A_{l}+\sum_{k} n_{k} D_{k}$. Let $g=g(C)$ be the geometric genus, and $\mu_{\text {tot }}(C)=\sum_{l} l \cdot m_{l}+\sum_{k} k \cdot n_{k}$ be the total Milnor number. Assume that $C$ is irreducible and $\mu_{\text {tot }}(C) \leq 5$. Then $g \geq 1$, and $g=1$ iff $\sum_{l} m_{l} A_{l}+\sum_{k} n_{k} D_{k}=5 A_{1}$.

Proof. The arithmetic genus of a quintic curve $C$ is 6 . Since $C$ is irreducible, each relevant singularity gives a genus drop of $\delta$ according to the following table

\begin{tabular}{|c|c|c|c|c|}
\hline Singularity & $A_{2 l-1}$ & $A_{2 l}$ & $D_{4}$ & $D_{5}$ \\
\hline$\delta$ & 1 & 1 & 3 & 3 \\
\hline
\end{tabular}


Assuming $C$ is as in the lemma, we get

$$
p_{a}(C)-p_{g}(C)=\sum_{l} m_{l} \delta\left(A_{l}\right)+\sum_{k} n_{k} \delta\left(D_{k}\right) \leq \sum_{l} m_{l} \cdot l+\sum_{k} n_{k} \cdot k \leq 5 .
$$

We thus obtain $p_{g}(C) \geq 1$, and the equality holds if and only if $C$ has $5 A_{1}$ singularities.

\subsection{Stratification of the dual variety $X^{\vee}$}

Motivated by Lemma 2.6, we will need to count the hyperplane sections $Y$ of $X$ that lead to 5 nodal plane quintics (via the projection from a very good line). In view of Proposition 2.4, this is equivalent to counting the 5 -tangent hyperplanes to a general cubic fourfold. This is part of a more general question regarding the structure of the dual variety $X^{\vee}$ that we briefly review below.

Let $X \subset \mathbb{P}^{5}$ be a general cubic fourfold. The dual variety $X^{\vee}$ is naturally stratified in terms of the singularities of the associated hyperplane section $Y=X \cap H_{b}$ (for $b \in X^{\vee} \subset B$ ). More precisely, $Y$ will have some combination of $A D$ singularities $R=\sum_{l} m_{l} A_{l}+\sum_{k} n_{k} D_{k}$ with $m_{l}, n_{k} \geq 0$, i.e. $Y$ has exactly $m_{l}$ singular points of type $A_{l}$ and $n_{k}$ singular points of type $D_{k}$. Prescribing a combination of singularities $R$ will define a stratum $\Sigma_{R}$ of $X^{\vee}$. In our set-up (i.e. $X$ a general cubic), we know (cf. Rem. 2.5) that at worst $A_{5}$ and $D_{5}$ occur and furthermore the codimension of the stratum $\Sigma_{R}$ associated to $R=\sum_{l} m_{l} A_{l}+\sum n_{k} D_{k}$ is

$$
\mu_{t o t}(R)=\sum_{l} l \cdot m_{l}+\sum_{k} k \cdot n_{k} \leq 5
$$

The versality property of Remark 2.5 easily allows one to determine the incidence of various strata (e.g. $\Sigma_{D_{4}} \subset \bar{\Sigma}_{3 A_{1}}$ ); we refer the interested reader to [7] for further discussion of the local structure of the strata $\Sigma_{R}$, and their geometric relevance. What is relevant here is to note that each $\bar{\Sigma}_{R}$ is a projective variety in $\left(\mathbb{P}^{5}\right)^{\vee}$ and thus has a degree $d_{R}$ and (expected) codimension $\mu_{\text {tot }}(R)$ (e.g. $d_{A_{1}}=\operatorname{deg}\left(X^{\vee}\right)=3 \cdot 2^{4}$ ). The com- 
putation of the degree $d_{R}$ is a classical question in enumerative geometry and singularity theory. The theory of Thom polynomials (Rimányi [28], Kazarian [16]) gives an effective method of computing the various $d_{R}$ as long as the simultaneous versal property (cf. Rem. 2.5) holds (in particular, the expected codimension $\mu_{\text {tot }}(R)$ is the actual codimension). For the low dimensional cases and small $\mu_{t o t}(R)$, Kazarian [16] gave explicit formulae. In particular, all that is needed for our purposes is the degree $\operatorname{deg}\left(\bar{\Sigma}_{5 A_{1}}\right)$, or equivalently the number of 5-tangent hyperplanes to a general cubic fourfold $X$.

Theorem 2.7 (Kazarian [16, Sect. 10]). Let $X$ be a general cubic fourfold. Then there are exactly 176,904 hyperplanes $H$ which are 5 tangent to $X$. Proof. The specific formula relevant to us is listed in [15, p. 6-7] (see "enum[4,5]" in loc. cit.). For the reader's convenience, we reproduce the formula for the number of 5 -tangent hyperplanes to a general degree $d \geq 3$ hypersuface in $\mathbb{P}^{5}$ :

$$
\begin{aligned}
m_{5 A_{1}}(d)= & \frac{1}{120}(d-2) d\left(d^{23}-18 d^{22}+154 d^{21}-832 d^{20}+3,181 d^{19}-9,332 d^{18}+23,306 d^{17}-56,258 d^{16}\right. \\
& +137,704 d^{15}-315,702 d^{14}+632,037 d^{13}-1,167,746 d^{12}+2,276,543 d^{11}-4,606,484 d^{10} \\
& +8,183,892 d^{9}-12,182,630 d^{8}+19,262,625 d^{7}-37,322,080 d^{6}+63,347,155 d^{5} \\
& \left.-72,821,310 d^{4}+73,475,394 d^{3}-156,527,928 d^{2}+284,455,368 d-193,415,040\right) .
\end{aligned}
$$

Setting $d=3$, we get $m_{5 A_{1}}(3)=176,904$ as claimed.

Remark 2.8. For comparison, we recall the situation for lower dimensional cubics. It is a standard fact that a cubic surface has 45 tritangent hyperplanes. For a general cubic threefold $Y$, there are $495\left(=2^{4}\left(2^{5}-\right.\right.$ 1) - 1) 4-tangent hyperplanes to $Y$. This can be obtained as a special case of Kazarian's results, or alternatively (and more geometrically), as the number of non-trivial odd theta characteristic for the intermediate Jacobian $J(Y)$. The latter claim follows by using the Prym description $J(Y)=\operatorname{Prym}(D, C)$ as above, and relating the 4-tangent hyperplanes to $Y$ to a certain configuration of conics relative to the quintic $C$ (which was 
studied in [31]). We note that $176,904=3^{5}\left(3^{6}-1\right)$, which indicates a relationship to the group of 3 -torsion points on an abelian variety, but we are not aware of a direct geometric link.

\section{The Euler characteristic of compactified Prym varieties}

The [21] model $Z=\overline{\mathcal{J}} / B$ of OG10 HK manifolds can be understood by means of relative compactified Prym varieties associated to double covers of plane quintics. Here, after a brief review of the compactified Prym varieties, we discuss the Euler characteristic of compactified Pryms. The main results (Prop. 3.1 and Cor. 3.4) are analogous to results of Beauville [3] for Jacobians.

\subsection{Compactified Prym varieties}

Let $f: D \rightarrow C$ be an étale double cover of irreducible locally planar curves and let $\iota: D \rightarrow D$ be the fixed point free involution associated to the covering. We denote by $J_{D}^{d}$ the degree $d$ generalized Jacobian of $D$ and by $\bar{J}_{D}^{d}$ its degree $d$ compactified Jacobian, parameterizing locally free and torsion free sheaves of rank 1 and degree $d$, respectively. We recall that since $D$ is irreducible with locally planar singularities, $\bar{J}_{D}^{d}$ is irreducible, and its smooth locus is precisely $J_{D}^{d}$ (e.g. [27]). We denote by $J_{D}$ and $\bar{J}_{D}$ the degree 0 generalized and compactified Jacobians. Notice, however, that because $D$ is irreducible, $\bar{J}_{D}^{d}$ is independent of the degree.

In $[21, \S 4]$ (cf. also $[1, \S 3]$ ), the compactified Prym variety is defined as the identity component of the fixed locus of the involution

$$
\begin{aligned}
-\iota^{*}: \bar{J}_{D} & \longrightarrow \bar{J}_{D} \\
F & \longmapsto\left(\iota^{*} F\right)^{\vee}:=\mathcal{H o m}_{\mathcal{O}_{D}}\left(\iota^{*} F, \mathcal{O}_{D}\right)
\end{aligned}
$$


acting on the degree zero compactified Jacobian of $D$. In formulae:

$$
\bar{P}_{D / C}:=\operatorname{Fix}^{\circ}\left(-\iota^{*}\right) \subset \bar{J}_{D}
$$

We refer the reader to $[21, \S 4]$ for more details on this construction. Let $g=p_{a}(C)$ be the arithmetic genus of $C$. By [21, Prop. 4.10 and Cor. 4.16], the compactified Prym variety $\bar{P}_{D / C}$ is an irreducible projective variety of dimension $g-1$. The open dense subset $P_{D / C}:=\bar{P}_{D / C} \cap J_{D}$ parameterizing line bundles, also called the generalized Prym variety, can be described in the following way. Let $\widetilde{D}$ and $\widetilde{C}$ be the normalization of the curves $D$ and $C$ respectively, and denote by $\widetilde{g}$ the genus of $\widetilde{C}$ so that $\widetilde{g}=g-\delta$ for some $\delta \geq 0$. There is a natural étale double cover $\widetilde{D} \rightarrow \widetilde{C}$ and $P_{D / C}$ fits in the exact sequence of algebraic groups

$$
0 \rightarrow G \rightarrow P_{D / C} \rightarrow P_{\widetilde{D} / \widetilde{C}} \rightarrow 0
$$

where $G$ is an affine group of dimension $\delta$ (a product of additive and multiplicative groups) and the Prym variety $P_{\widetilde{D} / \widetilde{C}}$ is a principally polarized abelian variety of dimension $\widetilde{g}-1$. More precisely, $G$ is isomorphic to $\mathcal{O}_{\widetilde{C}}^{*} /$ $\mathcal{O}_{C}^{*}=\oplus_{x \in \operatorname{Sing}(C)}\left(\mathcal{O}_{\widetilde{C}}^{*} / \mathcal{O}_{C}^{*}\right)_{x}$ and can be viewed inside $\mathcal{O}_{\widetilde{D}}^{*} / \mathcal{O}_{D}^{*} \cong G \times G$ with the anti-diagonal embedding.

It is well known that $J_{D}$ acts on $\bar{J}_{D}$ by tensorization. It is shown in [3, Lem 2.1] that the stabilizer of every point can be described in the following way. First recall that if $F$ is a rank one torsion free sheaf, then there is a partial normalization $n^{\prime}: D^{\prime} \rightarrow D$ and a torsion free sheaf $F^{\prime}$ on $D^{\prime}$, such that $\mathcal{E} n d_{\mathcal{O}_{D^{\prime}}}\left(F^{\prime}\right) \cong \mathcal{O}_{D^{\prime}}$ and $F=n_{*}^{\prime} F^{\prime}$. The curve $D^{\prime}$ is uniquely determined by the condition $D^{\prime}=\operatorname{Spec}_{\mathcal{O}_{D}} \mathcal{E} n d_{\mathcal{O}_{D}}(F)$. Moreover, if $\operatorname{deg} F=0$ and $\delta^{\prime}=p_{a}(D)-p_{a}\left(D^{\prime}\right)$, then $\operatorname{deg} F^{\prime}=-\delta^{\prime}$. Finally, by [3, Lem 3.1] the morphism

$$
n_{*}^{\prime}: \bar{J}_{D^{\prime}}^{-\delta^{\prime}} \rightarrow \bar{J}_{D}
$$

is an embedding. By [3, Lem. 2.1] the stabilizer of $F$ in $J_{D}$ is precisely the kernel of the pullback $\left(n^{\prime}\right)^{*}: J_{D} \rightarrow J_{D^{\prime}}$. By restriction, $P_{D / C}$ acts 
on $\bar{J}_{D}$ preserving $\operatorname{Fix}\left(-\iota^{*}\right)$, so there is an action of $P_{D / C}$ on $\bar{P}_{D / C}$. Let $F$ be a point in $\bar{P}_{D / C}$. An isomorphism $F \cong\left(\iota^{*} F\right)^{\vee}$, determines isomorphisms $\mathcal{E} n d_{\mathcal{O}_{D, x}} F_{x} \cong \mathcal{E}_{n d_{\mathcal{O}_{D, \iota x}}} F_{\iota(x)}$ for every $x \in D$ inducing an involution $\iota^{\prime}: D^{\prime} \rightarrow D^{\prime}$ naturally lifting $\iota$. The curve $C^{\prime}=D^{\prime} / \iota^{\prime}$ is a partial normalization of $C$ and there is a corresponding pullback map between Prym varieties

$$
\left(n^{\prime}\right)^{*}: P_{D / C} \rightarrow P_{D^{\prime} / C^{\prime}}
$$

The kernel of this morphism is naturally identified with $\mathcal{O}_{C^{\prime}}^{*} / \mathcal{O}_{C}^{*}$ and is precisely the stabilizer in $P_{D / C}$ of $F$.

\subsection{The Euler characteristic of compactified Pryms}

This proof of the following is an adaptation to compactified Pryms of the analogous statement by Beauville [3, Prop. 2.2].

Proposition 3.1. Let $f: D \rightarrow C$ and $\widetilde{g}$ be as above. If $\widetilde{g} \geq 2$ then $\chi\left(\bar{P}_{D / C}\right)=0$.

Proof. It is enough to show that for any integer $n \geq 2$ there is a free action of a group of order $n$ on $\bar{P}_{D / C}$. Indeed, this implies that $\chi\left(\bar{P}_{D / C}\right)$ is divisible by $n$ for every integer $n \geq 2$, and thus $\chi\left(\bar{P}_{D / C}\right)=0$. Consider the sequence (3.1). Since $G$ is a divisible (hence injective) abelian group, this sequence is split (as a sequence of abelian groups). It follows that as long as $P_{\widetilde{D} / \widetilde{C}}$ is an abelian variety of dimension $\geq 1$ (i.e. as long as $\widetilde{g} \geq 2$ ), we can find a group $K_{n}$ of order $n$ in $P_{D / C}$ which maps injectively to $P_{\widetilde{D} / \widetilde{C}}$. Since by the above discussion the stabilizer of any point of $\bar{P}_{D / C}$ is contained in the kernel of $P_{D / C} \rightarrow P_{\widetilde{D} / \widetilde{C}}$, it follows that $K_{n}$ acts freely on $\bar{P}_{D / C}$. This completes the proof.

We are now left with computing the Euler characteristic of the compactified Prym variety of an étale double cover of irreducible curves of geometric genus 1 . In view of Lemma 2.6 we only need to focus on nodal 
curves, so for the rest of this section we make the following assumption

(*) $C$ is a nodal curve.

We recalled earlier that for every partial normalization $n^{\prime}: D^{\prime} \rightarrow D$ there is a natural closed embedding

$$
n_{*}^{\prime}\left(\bar{J}_{D^{\prime}}^{-\delta^{\prime}}\right) \subset \bar{J}_{D}
$$

Notice the shift by $-\delta^{\prime}=-\left(p_{a}(D)-p_{a}\left(D^{\prime}\right)\right)$ in the degree. We wish to describe the intersection of $\bar{P}_{D / C}$ with each $n_{*}^{\prime}\left(\bar{J}_{D^{\prime}}^{-\delta^{\prime}}\right)$. We will do so expressing this intersection in terms of a "twisted" Prym. First, let us recall a few facts about relative duality applied to the finite morphism $n^{\prime}: D^{\prime} \rightarrow D$. Since $n^{\prime}$ is a finite morphism, it admits a relative dualizing sheaf which we denote by $\omega_{n^{\prime}}$. By relative duality

$$
\mathcal{H o m}_{\mathcal{O}_{D}}\left(n_{*}^{\prime} F^{\prime}, \mathcal{O}_{D}\right)=n_{*}^{\prime} \mathcal{H} o m_{\mathcal{O}_{D}}\left(F^{\prime}, \omega_{n^{\prime}}\right)
$$

Since $D$ and $D^{\prime}$ are nodal curves, their dualizing sheaves are locally free and $\omega_{D^{\prime}}=\omega_{n^{\prime}} \otimes\left(n^{\prime}\right)^{*} \omega_{D}$ is a line bundle on $D^{\prime}$ of degree $\operatorname{deg} \omega_{n^{\prime}}=-2 \delta^{\prime}$. There is a commutative diagram
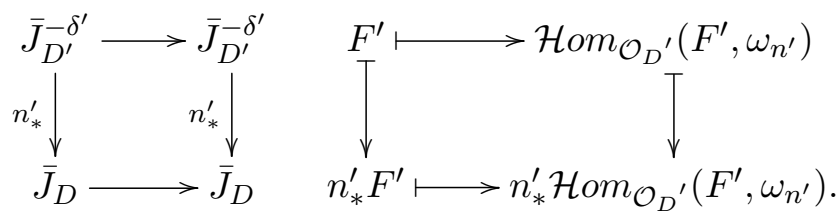

Proposition 3.2. Let $D \rightarrow C$ be an étale double cover of nodal and irreducible curves and let $D^{\prime} \rightarrow D$ be a partial normalization of $D$. Then $\bar{P}_{D / C} \cap n_{*}^{\prime}\left(\bar{J}_{D^{\prime}}^{-\delta^{\prime}}\right) \neq \emptyset$ if and only if there is an involution $\iota^{\prime}: D^{\prime} \rightarrow D^{\prime}$ lifting $\iota$. If this is the case, then

$$
\bar{P}_{D / C} \cap n_{*}^{\prime}\left(\bar{J}_{D^{\prime}}^{-\delta^{\prime}}\right) \cong \bar{P}_{D^{\prime} / C^{\prime}}
$$


where as above $C^{\prime}=D^{\prime} / \iota^{\prime}$ is a partial normalization of $C$.

Proof. We show that $\bar{P}_{D / C} \cap n_{*}^{\prime}\left(\bar{J}_{D^{\prime}}^{-\delta^{\prime}}\right)$ is the image under $n_{*}^{\prime}$ of a "twisted" Prym variety sitting in $\bar{J}_{D^{\prime}}^{-\delta^{\prime}}$. Let $F$ be a point in $\operatorname{Fix}\left(-\iota^{*}\right) \cap n_{*}^{\prime}\left(\bar{J}_{D^{\prime}}^{-\delta^{\prime}}\right)$ and let $F^{\prime}$ be such that $F=n_{*}^{\prime} F^{\prime}$. As observed above, this ensures that there is an involution $\iota^{\prime}: D^{\prime} \rightarrow D^{\prime}$, which lifts $\iota$. By uniqueness of the relative dualizing sheaf we see that $\left(\iota^{\prime}\right)^{*} \omega_{n^{\prime}} \cong \omega_{n^{\prime}}$ and hence

$$
\left(\iota^{*} F\right)^{\vee}=\iota^{*} \mathcal{H} \operatorname{Hom}_{\mathcal{O}_{D}}\left(n_{*}^{\prime} F, \mathcal{O}_{D}\right) \cong n_{*}^{\prime} \mathcal{H} o m_{\mathcal{O}_{D}}\left(\left(\iota^{\prime}\right)^{*} F^{\prime}, \omega_{n^{\prime}}\right) .
$$

Here, we have used that $\iota^{*}$ and $(\cdot)^{\vee}$ commute, since they commute on the dense open subset parametrizing locally free sheaves (similarly, $\left(\iota^{\prime}\right)^{*}$ and $\mathcal{H}_{o} m_{\mathcal{O}_{D^{\prime}}}\left(\cdot, \omega_{n^{\prime}}\right)$ commute); that for any sheaf $\mathcal{E}$ on $D, \iota^{*} \mathcal{E} \cong \iota_{*} \mathcal{E}$ (and similarly for $\iota^{\prime}$ ); and duality for finite morphisms (cf. Prop. 4.25 and Lem. 4.26 of [19]). It follows that, if $F \cong\left(\iota^{*} F\right)^{\vee}$, then $F^{\prime}$ is a fixed point of the involution

$$
\begin{aligned}
\tau_{D^{\prime}}: \bar{J}_{D^{\prime}}^{-\delta^{\prime}} & \longrightarrow \bar{J}_{D^{\prime}}^{-\delta^{\prime}} \\
F^{\prime} & \longmapsto \mathcal{H o m}_{\mathcal{O}_{D^{\prime}}}\left(\left(\iota^{\prime}\right)^{*} F^{\prime}, \omega_{n^{\prime}}\right) .
\end{aligned}
$$

This implies that

$$
\operatorname{Fix}\left(-\iota^{*}\right) \cap n_{*}^{\prime}\left(\bar{J}_{D^{\prime}}^{-\delta^{\prime}}\right)=\operatorname{Fix}(\tau)
$$

Since $\left(\iota^{\prime}\right)^{*} \omega_{n^{\prime}} \cong \omega_{n^{\prime}}$ it is not hard to see (e.g. [24, p. 329]) that there exists a degree $\delta^{\prime}$ line bundle $L$ on $D^{\prime}$ such that

$$
\omega_{n^{\prime}} \cong L^{\vee} \otimes\left(\iota^{\prime}\right)^{*} L^{\vee}
$$

This shows that under the isomorphism $\bar{J}_{D^{\prime}}^{-\delta^{\prime}} \rightarrow \bar{J}_{D^{\prime}}$ defined by tensoring with $L$, we have an isomorphism

$$
\operatorname{Fix}\left(-\left(\iota^{\prime}\right)^{*}\right) \cong \operatorname{Fix}(\tau) \text {. }
$$

Now by $\left[21\right.$, Cor. 4.16] both $\operatorname{Fix}\left(-\iota^{*}\right)$ and $\operatorname{Fix}\left(-\left(\iota^{\prime}\right)^{*}\right) \cong \operatorname{Fix}(\tau)$ have ex- 
actly four irreducible connected components which are isomorphic to each other. The compactified Prym variety is the one containing the identity, and the isomorphism of any component $Z$ with the Prym is defined by tensorization with a line bundle belonging to $Z$ (cf. [21, (4.8)]). This shows that this isomorphism preserves the local type of sheaves and hence that every component has the same strata appearing. Now look at (3.2). The right hand side has 4 connected components and hence so has the left hand side. By the discussion above, if one component intersects $n_{*}^{\prime}\left(\bar{J}_{D^{\prime}}^{-\delta^{\prime}}\right)$ then so do all the others. In particular, each component of Fix $\left(-\iota^{*}\right)$ intersects $n_{*}^{\prime}\left(\bar{J}_{D^{\prime}}^{-\delta^{\prime}}\right)$ in a connected closed subset which has to be isomorphic to $\bar{P}_{D^{\prime} / C^{\prime}}$.

Remark 3.3. Without assuming that $C$ (and $D$ ) are nodal, the same conclusion holds true for any stratum corresponding to a partial normalization $D^{\prime}$ that is also locally planar.

Corollary 3.4. If $C$ is a nodal curve of geometric genus 1 then the Euler characteristic $\chi\left(\bar{P}_{D / C}\right)=1$.

Proof. Under the assumption, $\bar{J}_{D}$ admits a stratification in generalized Jacobians of partial normalizations of $D$. The stratification is indexed by the subset of the set $A$ of nodes of $D$ in the following way. There is a natural action of $\iota$ on $A$, so we can talk of $\iota$-invariant subsets of $A$. For every subset $B \subset A$, the stratum corresponding to the normalization $D_{B}$ of $D$ at the nodes of $B$ is isomorphic to

$$
0 \rightarrow\left(\mathbb{C}^{*}\right)^{\# A \backslash B} \rightarrow J_{D} \rightarrow J_{\widetilde{D}} \rightarrow 0 .
$$

By the proposition above, such a stratum intersects the compactified Prym variety if and only if $B$ is $\iota$-invariant. If this is the case, then the induced stratum on the Prym is given by

$$
0 \rightarrow\left(\mathbb{C}^{*}\right)^{(\# A \backslash B) / \iota} \rightarrow P_{D / C} \rightarrow P_{\widetilde{D} / \widetilde{C}}=\{p t\}
$$

Every stratum has trivial Euler number, except for the one corresponding 
to $B=A$, which is just one point.

\section{Completion of the proof of Theorem 1.1}

By the discussion of Section 2 and Proposition 3.1, the only contribution to the Euler characteristic is due to compactified Pryms $\bar{P}_{D / C}$, where $C$ is an irreducible plane quintic of geometric genus 1. By Lemma 2.6 and Proposition 2.4, such curves arise via projections from a general line $\ell$ on a 5-nodal hyperplane section $Y=X \cap H$. By Theorem 2.7, there are 176, 904 such hyperplanes. Finally, by Corollary 3.4, the contribution of each such hyperplane is 1 . By Corollary 2.2, we conclude $\chi(Z)=\chi(\overline{\mathcal{J}})=$ $176,904 \cdot 1$.

\section{References}

[1] E. Arbarello, G. Saccà, and A. Ferretti, Relative Prym varieties associated to the double cover of an Enriques surface, J. Differential Geom. 100 (2015), no. 2, 191-250.

[2] A. Beauville, Variétés Kähleriennes dont la première classe de Chern est nulle, J. Differential Geom. 18 (1983), no. 4, 755-782 (1984).

[3] _ Counting rational curves on K3 surfaces, Duke Math. J. 97 (1999), no. 1, 99-108.

[4] C. H. Clemens and P. A. Griffiths, The intermediate Jacobian of the cubic threefold, Ann. of Math. (2) 95 (1972), 281-356.

[5] S. Casalaina-Martin, S. Grushevsky, K. Hulek, and R. Laza, Complete moduli of cubic threefolds and their intermediate Jacobians, Proc. Lond. Math. Soc 101112/plms.12375.

[6] S. Casalaina-Martin and R. Laza, The moduli space of cubic threefolds via degenerations of the intermediate Jacobian, J. Reine Angew. Math. 633 (2009), 29-65. 
[7] _ Simultaneous semi-stable reduction for curves with ADE singularities, Trans. Amer. Math. Soc. 365 (2013), no. 5, 2271-2295.

[8] S. E. Cappell, L. G. Maxim, and J. L. Shaneson, Euler characteristics of algebraic varieties, Comm. Pure Appl. Math. 61 (2008), no. 3, 409421.

[9] M. de Cataldo, A. Rapagnetta, and G. Saccà, The Hodge numbers of O’Grady 10 via Ngô strings, arXiv:1905.03217, 2019.

[10] R. Donagi and E. Markman, Spectral covers, algebraically completely integrable, Hamiltonian systems, and moduli of bundles, Integrable systems and quantum groups (Montecatini Terme, 1993), Lecture Notes in Math., vol. 1620, Springer, Berlin, 1996, pp. 1-119.

[11] W. Fulton, Introduction to toric varieties, Annals of Mathematics Studies, vol. 131, Princeton University Press, Princeton, NJ, 1993, The William H. Roever Lectures in Geometry.

[12] M. Green, Y.J. Kim, R. Laza, and C. Robles, The LLV decomposition of hyper-Kaehler cohomology, arXiv:1906.03432, 2019.

[13] L. Goettsche, The Betti numbers of the Hilbert scheme of points on a smooth projective surface, Math. Ann. 286 (1990), no. 1-3, 193-207.

[14] L. Goettsche and W. Soergel, Perverse sheaves and the cohomology of Hilbert schemes of smooth algebraic surfaces, Math. Ann. 296 (1993), no. 2, 235-245.

[15] M. 亡̀. Kazarian, Enumeration of tangencies of k-planes with a hypersurface in the projective $n$-spac, http://www.miras.ru/ kazarian/Wnmath/Enumleg4.ps.

[16] - Multisingularities, cobordisms, and enumerative geometry, Uspekhi Mat. Nauk 58 (2003), no. 4(352), 29-88. 
[17] J. Kollár, R. Laza, G. Saccà, and C. Voisin, Remarks on degenerations of hyper-Kähler manifolds, Ann. Inst. Fourier (Grenoble) 68 (2018), no. 7, 2837-2882.

[18] R. Laza, The moduli space of cubic fourfolds via the period map, Ann. of Math. (2) 172 (2010), no. 1, 673-711.

[19] Q. Liu, Algebraic geometry and arithmetic curves, Oxford Graduate Texts in Mathematics, vol. 6, Oxford University Press, Oxford, 2002.

[20] E. Looijenga and V. A. Lunts, A Lie algebra attached to a projective variety, Invent. Math. 129 (1997), no. 2, 361-412.

[21] R. Laza, G. Saccà, and C. Voisin, A hyper-Kähler compactification of the intermediate Jacobian fibration associated with a cubic 4-fold, Acta Math. 218 (2017), no. 1, 55-135.

[22] S. Mozgovyy, The Euler number of O'Gradys 10-dimensional symplectic manifold, Ph.D. thesis, Johannes Gutenberg-Universität Mainz, 2007.

[23] G. Mongardi, A. Rapagnetta, and G. Saccà, The Hodge diamond of O'Grady's six-dimensional example, Compos. Math. 154 (2018), no. 5, 984-1013.

[24] D. Mumford, Prym varieties. I, pp. 325-350, Academic Press, New York, 1974.

[25] K. G. O'Grady, Desingularized moduli spaces of sheaves on a K3, J. Reine Angew. Math. 512 (1999), 49-117.

[26] A. Rapagnetta, On the Beauville form of the known irreducible symplectic varieties, Math. Ann. 340 (2008), no. 1, 77-95.

[27] C. J. Rego, The compactified Jacobian, Ann. Sci. École Norm. Sup. (4) 13 (1980), no. 2, 211-223. 
[28] R. Rimányi, Thom polynomials, symmetries and incidences of singularities, Invent. Math. 143 (2001), no. 3, 499-521.

[29] S. M. Salamon, On the cohomology of Kähler and hyper-Kähler manifolds, Topology 35 (1996), no. 1, 137-155.

[30] M. Verbitsky, Cohomology of compact hyper-Kähler manifolds and its applications, Geom. Funct. Anal. 6 (1996), no. 4, 601-611.

[31] F. P. White, On the 5-Tangent Conics of a Plane Quintic Curve, Proc. London Math. Soc. (2) 30 (1930), no. 5, 347-358.

Klaus Hulek

Institut für Algebraische Geometrie

Leibniz Universität Hannover

30060 Hannover, Germany

Email: hulek@math.uni-hannover.de

Radu Laza

Stony Brook University

Stony Brook, NY 11794, USA

Email: radu.laza@stonybrook.edu

Giulia Saccà

Columbia University

New York, NY 10027, USA

Email: gs3032@columbia.edu 